\title{
Morten Nøjgaard, Temps, réalisme et description
}

\section{Luigi Luison}

\section{Q OpenEdition}

\section{Journals}

\section{Edizione digitale}

URL: http://journals.openedition.org/studifrancesi/31056

DOI: 10.4000/studifrancesi.31056

ISSN: 2421-5856

\section{Editore}

Rosenberg \& Sellier

\section{Edizione cartacea}

Data di pubblicazione: 1 avril 2006

Paginazione: 219-220

ISSN: 0039-2944

\section{Notizia bibliografica digitale}

Luigi Luison, «Morten Nøjgaard, Temps, réalisme et description», Studi Francesi [Online], 148 (XLX |I) | 2006, online dal 30 novembre 2015, consultato il 19 avril 2021. URL: http://journals.openedition.org/ studifrancesi/31056 ; DOI: https://doi.org/10.4000/studifrancesi.31056

\section{Questo documento è stato generato automaticamente il 19 avril 2021.}

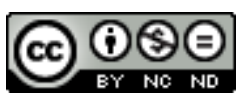

Studi Francesi è distribuita con Licenza Creative Commons Attribuzione - Non commerciale - Non opere derivate 4.0 Internazionale. 


\title{
Morten Nøjgaard, Temps, réalisme et description
}

\author{
Luigi Luison
}

\section{NOTIZIA}

MORTEN NøJGAARD, Temps, réalisme et description, Paris, Editions Champions, 2004, pp. 252.

1 Nel Novecento la critica letteraria ha scoperto l'importanza dei tratti caratteristici del discorso narrativo per capire il funzionamento del romanzo. Sono state esplorate e messe in evidenza tutte le tecniche che servono a creare i meccanismi dinamici dell'azione romanzesca; e quella che oggi chiamiamo "narratologia" si può considerare a tutti gli effetti una vera e propria scienza. Per chi si occupa di studi letterari, alcune nozioni come "focalizzazione" o "autore implicito" sono ormai di uso quotidiano. L'interesse suscitato dalla "meccanica" del racconto, però, ha fatto un po' dimenticare le basi filosofiche che determinano i tratti distintivi del discorso narrativo.

2 È proprio in questo contesto ontologico del racconto che si inseriscono i primi due saggi del volume, intitolati Le temps de la fiction: la dimension existentielle de la narrativité $\mathrm{e}$ Le réalisme littéraire: une approche phénoménologique. Attraverso questi due lavori, l'A. si propone di perseguire due principali obiettivi: in primo luogo individuare ed evidenziare la base ontologica del dinamismo romanzesco per poter approfondire la nozione di tempo narrativo, ed in secondo luogo mostrare come questa temporalità viene considerata una condizione epistemologica utile all'uomo per potersi collocare in relazione al suo tempo. In particolare, l'A. focalizza la sua attenzione sull'elemento distintivo della temporalità romanzesca, considerato da lui stesso come base fondamentale di partenza per ogni tipo di studio avviato al raggiungimento di una comprensione delle dinamiche del realismo letterario.

3 L'A., partendo dalla convinzione che la finalità ultima del racconto è di dare una risposta ai problemi che si pone l'uomo sulla sua esistenza nel tempo, vuole dimostrare 
come la creazione di una temporalità vissuta sia un tratto costitutivo della letteratura narrativa, nonostante essa sia stata poco studiata nel suo contesto filosofico.

4 Procedendo con le sue riflessioni sulla natura della temporalità narrativa, l'A. dimostra, inoltre, che lo studio del tempo della finzione permette di gettare uno sguardo nuovo sul tema tanto dibattuto del realismo letterario, proprio grazie alla dimensione esistenziale che viene riconosciuta a tale temporalità.

5 Il volume si chiude con il terzo saggio dal titolo Comment décrire la description?: technique narrative et espace littéraire. Con questo lavoro l'A. prosegue ed amplia le ricerche sulla descrizione letteraria effettuate in questi ultimi anni da parte di studiosi dell'argomento, in particolare quelle di Philippe Hamon, le cui opere hanno profondamente influenzato, secondo l'A., l'odierna analisi della descrizione letteraria. Attraverso questo saggio molto più tecnico rispetto ai primi due, l'A. porta avanti la riflessione teorica e filosofica in essi iniziata; riflessione finalizzata sia all'esplorazione della fenomenologia della descrizione, sia ai modi ontologici dello spazio letterario.

6 Con questo volume l'A. intende fornire uno studio esaustivo delle tecniche descrittive, e un modello pratico per l'analisi della descrizione letteraria. 